\title{
Multi-level Influences on Breast Cancer Screening in Primary Care
}

\author{
Tracy Onega, $P h D, M A, M S^{1,2,3}$, Tor D. Tosteson, $S c D^{1,2,3}$, Julie Weiss, $M S^{7}$, Jennifer S. Haas, $M D, M S^{4,5,6}$, \\ Martha Goodrich, $M S^{1,2}$, Roberta DiFlorio, $M D^{7}$, Charles Brackett, MD, MPH', Cheryl Clark, MD, SCD , \\ Kimberly Harris, $M M^{4}$, and Anna N. A. Tosteson, $S C D^{2,3}$
}

'Department of Biomedical Data Science, Geisel School of Medicine at Dartmouth, Lebanon, NH, USA; ${ }^{2}$ Norris Cotton Cancer Center, Geisel
School of Medicine at Dartmouth, Lebanon, NH, USA; ${ }^{3}$ The Dartmouth Institute for Health Policy and Clinical Practice, Geisel School of Medicine at
Dartmouth, Lebanon, NH, USA; ' ${ }^{4}$ ivision of General Internal Medicine and Primary Care, Brigham and Women's Hospital, Boston, MA, USA;
${ }^{5}$ Harvard Medical School, Boston, MA, USA; ${ }^{6}$ Harvard T.H. Chan School of Public Health, Boston, MA, USA; ${ }^{7}$ Department of Radiology, Dartmouth-
Hitchcock Medical Center, Lebanon, NH, USA; ${ }^{8}$ Department of General Internal Medicine, Dartmouth-Hitchcock Medical Center, Lebanon, NH, USA.

BACKGROUND: Use of breast cancer screening is influenced by factors associated with patients, primary care providers, practices, and health systems.

OBJECTIVE: We examined the relative effects of these nested levels on four breast cancer screening metrics. DESIGN: Aweb-based survey was completed at 15 primary care practices within two health systems representing 306 primary care providers (PCPs) serving 46,944 women with a primary care visit between 1/2011-9/2014. Analyses occurred between $1 / 2017$ and 5/2017.

MAIN MEASURES: Across four nested levels (patient, PCP, primary care practice, and health system), frequency distributions and adjusted rates of primary care practice characteristics and survey results for four breast screening metrics (percent screened overall, and percent screened age 40-49, 50-74, and 75+) were reported. We used hierarchical multi-level mixed and random effects analysis to assess the relative influences of $\mathrm{PCP}$, primary care practice, and health system on the breast screening metrics.

KEY RESULTS: Overall, the proportion of women undergoing breast cancer screening was 73.1\% (73.4\% for ages 40-49, 76.5\% for 50-74, and 51.1\% for 75+). Patient ethnicity and number of primary care visits were strongly associated with screening rates. After adjusting for woman-level factors, $24 \%$ of the overall variation among PCPs was attributable to the primary care practice level, $35 \%$ to the health system level, and $41 \%$ to the residual variation among PCPs within practice. No specific provider-level characteristics were found to be statistically significant determinants of screening rates.

CONCLUSIONS: After accounting for woman-level characteristics, the remaining variation in breast cancer screening was largely due to provider and health system variation.

KEY WORDS: breast cancer screening; primary care practice breast screening.

J Gen Intern Med 33(10): 1729-37

DOI: $10.1007 / \mathrm{s} 11606-018-4560-1$

(C) Society of General Internal Medicine 2018

Prior Presentation The Australasian Society for Breast Disease 11th Scientific Meeting. Gold Coast. October 5-7, 2017.

Received November 15, 2017

Revised April 24, 2018

Accepted June 28, 2018

Published online August 3, 2018

\section{BACKGROUND}

Although breast cancer screening takes place in radiology clinics, primary care providers (PCPs) and primary care practices, hereafter referred to as "practices," are largely responsible for ordering and tracking screening among eligible patient populations. Tracking captures women's adherence to evidence-based screening regimens, including age of screening initiation and cessation, and screening frequency. These measures are intended to support delivery of quality care and are used for population management, accountability metrics, and provider information. Given the heterogeneity of breast cancer screening guidelines, patient education, communication, risk assessment, and shared decisionmaking are also important components of breast cancer screening. Several conceptual models and frameworks related to breast cancer screening have been published (1-5), all of which underscore the importance of processes of care and the multi-level nature of breast cancer screening delivery.

Factors at the patient, PCP, practice, and health system levels are thought to influence patterns of breast cancer screening use, although the roles of specific factors have not been well investigated. Further, the relative influence of each of these levels on breast cancer screening measures is unknown. Prior work has shown marked variation among practices for breast cancer screening patterns $(3,6)$. In our earlier study of 13 practices, the practice-level proportions of women initiating breast cancer screening at age 40 varied from 54.3 to $84.8 \%$, and those continuing screening beyond age 75 varied from 37.2-78.3\% (3). Similarly, we found variation among PCP survey respondents $(N=385)$ in processes of care related to breast cancer screening, such as use of electronic health record (EHR)-based decision support and automated reports of patients overdue for screening (7). At the woman level, a host of factors are reported to be associated with greater breast cancer screening guideline adherence, including higher educational attainment and no comorbidities (8). Less is known about effects at the health system level, although some evidence suggests healthcare system-level influences. For example, healthcare systems that adopted specific models of healthcare delivery, such as patient-centered medical homes (PCMH), improved in some screening metrics, but not in 
others, suggesting a system-level effect, albeit heterogeneous (9). Thus, patient, PCP, practice, and healthcare system-level effects seem to influence breast cancer screening utilization patterns measured from a primary care perspective.

Specific processes of care or structural characteristics of practices and healthcare systems have been hypothesized as important for breast cancer screening $(10,11)$. Important structural characteristics may include practice size, provider composition, and type. Healthcare system-level characteristics that may influence screening patterns include staffing ratios of generalists to specialists, facilities/resources, including EHR systems and reminders, importance of quality measures, perceived commitment to service, and cost. Processes of care are healthcare-related activities performed for, on behalf of, or by a patient (12). For breast cancer screening, process measures can include elements such as type of patient and PCP reminders about screening and use of a breast cancer risk calculator. The objective of this study was to examine the effect of PCP, practice, and health system-level characteristics and processes on the breast cancer screening metrics: overall percent screened, percent of 40-49-year-olds screened, percent screened (ages 50-74 years - included in all guidelines), and percent screening past age 75 years, for which guidelines suggest weighing the harms and benefits of continued screening while accounting for women-level characteristics. Using a multi-level modeling approach, we sought to estimate the relative influences of PCPs, practices, and systems on the breast screening metrics.

\section{METHODS}

\section{Study Population and Setting}

We conducted this study in a breast cancer screening-focused Population-based Research Optimizing Screening for Personalized Regimens (PROSPR) Research Center (PRC) (1, 13, 14). Our PROSPR PRC included the primary care populations of the Dartmouth-Hitchcock regional network in New Hampshire and the Brigham and Women's Hospital primary care network in greater-Boston. Practice Managers at 15 (of 25) practices completed a survey (detailed below), representing 306 providers (physicians, physician assistants, and nurse practitioners) affiliated with those practices. The study period was $1 / 1 / 2011-9 / 30 / 2014$, and all women in the cohort had 27 months of observation. Analyses occurred between 1/2017 and $5 / 2017$. Therefore, the women's primary care visit occurred in the first half of the study period, up to 6/30/2012, to allow enough follow-up time for observation. Women were eligible $(N=46,944)$ if they had at least one visit to one of the 15 practices that completed the survey, were 40-89 years of age, and had no documented history of breast cancer before or during the study period. The study was approved by the Institutional Review Boards (IRB) of Dartmouth College and Partners Healthcare.

\section{MAIN MEASURES}

\section{Primary Care Practice Survey}

Our PRC and clinical partners developed a 22 -item practice survey. The survey was web-based, targeted to practice managers who were instructed to consult with their practice's medical director as needed, and a $\$ 50$ Amazon gift-card incentive was provided. The survey was deployed between December 2014 and March 2015. Of the 25 practices invited to participate in the survey, $15(60 \%)$ completed it with no missing responses. The survey content consisted of the structural elements (number of items in parentheses): appointment length (1), interactive patient portal (1), patient population size (1), and provider composition (1); and the process domains: screening guidelines (3), risk assessment (3), communication (5), decision support (1), and documentation (5), along with a final question (1) recording the role/title of the person(s) completing the survey.

\section{Key Variables}

Woman Level. Sociodemographic and clinical data are routinely collected for the women in our healthcare system through a variety of electronic data sources, including the electronic health record (EHR), radiology information systems, and billing data. We recorded age at study entry in categories $(40-49,50-59$, $60-69,70-79,80+$ ) and self-reported race and ethnicity (nonHispanic White, non-Hispanic Black, non-Hispanic Asian/ Pacific Islander, Hispanic, and other/unknown). We collected insurance status categorized as: private, Medicare, Medicaid, other, and uninsured. The number of primary care visits for each woman within a calendar year was calculated, as was the number of comorbidities, based upon the Charlson comorbidity index (15).

Provider Level. Provider characteristics were taken from the administrative data for the respective health systems. Specifically, we recorded the health system with which the providers were affiliated (Dartmouth-Hitchcock or Brigham and Women's Hospital), and the type of provider (physician, nurse practitioner, or physician assistant). Additionally, the number of women per provider was computed.

Practice Level. We included structural and process characteristics of the practices that completed the survey. We assessed full-time equivalency (FTE) for each of the following: medical assistants/nurses, physician assistants, nurse practitioners, and physicians. We collected appointment time (minutes) for a preventive care or annual visit; use of breast screening measures for reporting; estimated percent of patients using a patient portal; whether there was documentation - and form thereof - for patients who declined screening; and if there was a structured field for documenting provider discussions of screening. Questions related to use of screening guidelines included the following: use of a nationally recognized set of guidelines (e.g., United States Preventive Services Task Force, 
American College of Radiology, and American Cancer Society) typical age for breast cancer screening initiation endorsed by the practice (if any), typical stopping age for breast cancer screening (if any), and typical screening interval (1 or 2 years). We collected data on use of reminders that breast cancer screening is due, such as patient reminders by verbal prompt from PCP, patient reminders handled by radiology, and multiple reminders (quantity) to patient via mail, phone, EHR, or e-mail. Practices recorded use of breast cancer risk assessment tools and whether a risk calculator was embedded in their EHR. The number of women within each practice was calculated for practice study population size. We compared women's characteristics (age, race/ethnicity, insurance, primary care visits, comorbidities) between the practices responding to the survey, and those not, and found no notable differences except a lower proportion of nonHispanic Black women (7.0\% in included practices, $4.3 \%$ in excluded) and higher proportion of Hispanic women in the excluded practices (5.0 vs. $10.3 \%)$.

Outcome-Breast Cancer Screening. We defined a breast cancer screening event in the observation period, if the woman had any mammogram (digital mammography or digital breast tomosynthesis) with bilateral views within 27 months following her first primary care visit in our study. The women were divided into three screening groups (40-49, $50-74$, and $75+$ ) based on her age at her primary care visit.

\section{Statistical Analyses}

We examined the distributions $(N, \%)$ of structural characteristics across all three of the nested levels (woman, PCP, practice). To compute the adjusted rates of the practice characteristics and processes for the four breast cancer screening metrics (percent screened overall, percent screened 40-49, percent screened 50 74 , and percent screened 75+) within the nested levels, generalized linear mixed effects regression models (including both fixed and random effects) were implemented, including the women and PCP characteristics as potential confounders. We report adjusted rates and confidence intervals according to practice characteristics or processes and present $p$ values for comparisons between categories.

To understand the relative influences of PCP, and practice, and health system factors on the four screening outcomes measured, we performed a variance components analysis using estimates from the generalized linear mixed model estimate the variation in the log odds of screening explained by each level (PCP, practice, and system) after adjusting for woman-level characteristics.

\section{KEY RESULTS}

\section{Study Population Characteristics}

The study population included 15 practices, 306 providers and 46,944 women age 40+ who were associated with those PCPs and practices. About half of the practices had $<9$ FTE physicians $(N=8), 20-30$-min appointment times for preventive/ routine care visits $(N=8)$, documented whether a patient declined screening in notes only, and had an overall practice study population size of $\leq 8000$ patients (Table 1). The majority of practices reported no structured documentation of PCPs discussing breast cancer screening $(N=10)$, and reported $\leq$ $50 \%$ of their patients using an interactive patient portal $(N=9)$ (Table 1). The vast majority of providers affiliated with the 15 practices were physicians (87\%; $4 \%$ NPs or PAs, and $9 \%$ unknown). Most women were between 40 and 69 years of age $(86.7 \%)$, were non-Hispanic Whites $(81.4 \%)$, had private insurance $(68 \%)$, and had no comorbidities $(74 \%)$ (Table 1).

\section{Structural and Process Characteristics of Practices}

Almost none of the practices $(N=2)$ endorsed stopping breast cancer screening at age 75, and just over half $(N=8)$ endorsed initiating screening at age 40. (Table 2) Fewer than half $(N=7)$ of the practices reported use of a risk assessment tool, with almost $75 \%$ reporting lack of a breast cancer risk calculator embedded in the EHR. Most $(N=12)$ practices reminded patients verbally at the time of a visit if they were due for breast cancer screening, and just over half noted that patient reminders are handled by the radiology department(s) (Table 2).

\section{Breast Cancer Screening Outcome Measures}

The overall percent of women who had received breast cancer screening was $73.1 \%$ (95\% CI) and by age groups: ages 40 49, 73.4\% (95\% CI); ages 50-74, 76.5\% (95\% CI); ages 75+, $51.1 \%$ (95\% CI). The proportion of women screened overall, and by age groups in relation to practice-level processes, revealed no significant differences by any process measure for any of the breast cancer screening metrics measured (Table 3). Screening percent for women ages 40-49 varied significantly by provider type $(p=0.02)$, although that difference may have been driven largely by the "unknown" category (Table 4). Adjusted rates for all of the breast cancer screening metrics varied significantly by woman-level characteristics. For example, Hispanic women had a significantly higher $(p<0.0001)$ percent screened overall, for women ages $40-49,50-74$, and $75+$ compared to women of all other racial/ ethnic categories (Table 4). For women ages 40-49 and 50 74 , more PCP visits was significantly related to a higher percent screened (Table 4).

\section{Components of Variation in Breast Screening Outcomes}

When evaluating the relative influence of PCP-, practice-, and health system levels on the observed variation in the breast cancer screening metrics, we found that after adjusting for woman-level factors, $41 \%$ of the overall remaining variation 
Table 1 Structural Characteristics of Women Age 40+, Primary Care Providers (PCPs), and Practices Represented Within a PROSPR Research Center Study Population

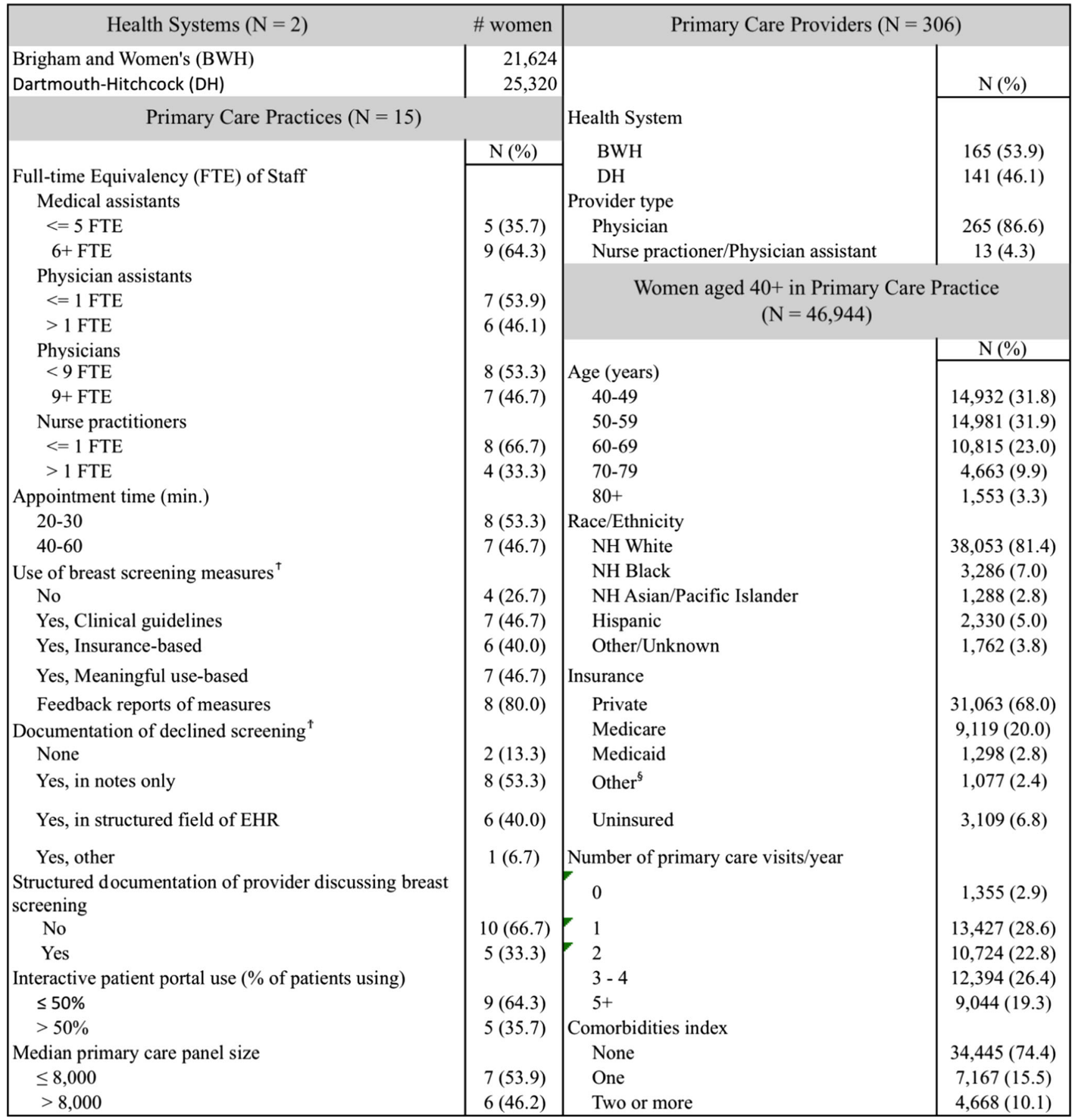

PROSPR population-based research optimizing screening through personalized regimens, BWH Brigham and Women's Hospital, DH DartmouthHitchcock, FTE full-time equivalent, PCP primary care physician

Missing (N): medical assistants FTE (1), physician assistants FTE (2), nurse practitioners FTE (3), interactive patient portal (1), racelethnicity (225), insurance (1278), comorbidity score (664)

*Not mutually exclusive

†Other insurance defined as other federal or state health insurance programs or not otherwise specified

was attributable to PCPs, $24 \%$ to the practice level and $35 \%$ to the health system level (Fig. 1).

For breast screening among women ages 40-49, most of the variation was at the health system level (62\%), while $23 \%$ was attributable to PCPs, and $15 \%$ to practices (Fig. 1).
Residual variation in screening for women ages 50-74 was greatest at the PCP level (45\%), with $36 \%$ at the practice level, and $19 \%$ at the system level. For percent screened, ages $75+$, most of the residual variability was among PCPs within practices $(56 \%$ ), with $20 \%$ due to the practice level, and $24 \%$ to the 
Table 2 Process Characteristics of Primary Care Practices for the PROSPR Research Center Study Population

\begin{tabular}{|c|c|c|c|c|c|c|c|}
\hline & $\begin{array}{c}\text { Primary Care } \\
\text { Practices } \\
(\mathrm{N}=15)\end{array}$ & $\begin{array}{l}\text { Primary Care } \\
\text { Providers (N } \\
=306)\end{array}$ & $\begin{array}{c}\text { Women }(40+\text { yrs. }) \\
\text { in Primary Care } \\
(\mathrm{N}=46,944)\end{array}$ & \multicolumn{2}{|c|}{$\begin{array}{c}\text { Median Number of Women at the } \\
\text { Primary Care Practice Level }\end{array}$} & \multicolumn{2}{|c|}{$\begin{array}{l}\text { Median Number of Women at the } \\
\text { Primary Care Provider Level }\end{array}$} \\
\hline Facility Screening Practices & \multicolumn{3}{|c|}{$\mathrm{N}(\%)$} & $\mathrm{N}$ (practice) & Median Women (IQR) & $\mathrm{N}$ (Providers) & Median Women (IQR) \\
\hline \multicolumn{8}{|l|}{ Intiation age for screening: 40 years } \\
\hline No & $7(46.7)$ & $156(51.0)$ & $23,116(49.2)$ & 7 & $3,429(395-5,751)$ & 156 & $47(15-250)$ \\
\hline Yes & $8(53.3)$ & $150(49.0)$ & $23,828(50.8)$ & 8 & $1,832(1,081-5,078)$ & 150 & $47(12-282)$ \\
\hline \multicolumn{8}{|c|}{ Intiation age for screening: by provider's choice } \\
\hline No & $10(66.7)$ & $247(80.7)$ & $36,080(76.9)$ & 10 & $3,561(1,573-5,751)$ & 247 & $38(14-241)$ \\
\hline Yes & $5(33.3)$ & $59(19.3)$ & $10,864(23.1)$ & 5 & $2,370(395-3,429)$ & 59 & $77(13-367)$ \\
\hline \multicolumn{8}{|l|}{ Stopping age for screening: 75 years } \\
\hline No & $13(86.7)$ & $207(67.6)$ & $32,604(69.4)$ & 13 & $2,044(589-4,330)$ & 207 & $51(13-282)$ \\
\hline Yes & $2(13.3)$ & $99(32.4)$ & $14,340(30.6)$ & 2 & $7,170(6,501-7,839)$ & 99 & $37(14-155)$ \\
\hline \multicolumn{8}{|c|}{ Stopping age for screening: provider's choice } \\
\hline No & $3(20.0)$ & $114(37.3)$ & $20,091(42.8)$ & 3 & $6,501(571-7,839)$ & 114 & $49(15-265)$ \\
\hline Yes & $12(80.0)$ & $192(62.3)$ & $26,853(57.2)$ & 12 & $1,832(492-3,880)$ & 192 & $47(12-253)$ \\
\hline \multicolumn{8}{|l|}{ Screening interval: 1 year } \\
\hline No & $7(46.7)$ & $156(51.0)$ & $21,306(45.4)$ & 7 & $2,370(395-5,751)$ & 156 & $47(15-206)$ \\
\hline Yes & $8(53.3)$ & $150(49.0)$ & $25,638(54.6)$ & 8 & $2,737(1,081-5,079)$ & 150 & $47(12-305)$ \\
\hline \multicolumn{8}{|l|}{ Screening interval: provider's choice } \\
\hline No & $8(53.3)$ & $161(52.6)$ & $29,816(63.5)$ & 8 & $4,254(1,317-5,415)$ & 161 & $49(13-310)$ \\
\hline Yes & $7(46.7)$ & $145(47.4)$ & $17,128(36.5)$ & 7 & $1,619(395-4,330)$ & 145 & $46(14-156)$ \\
\hline \multicolumn{8}{|c|}{$\begin{array}{l}\text { Patient reminders with verbal prompt from } \\
\text { provider/care team during office visit }\end{array}$} \\
\hline No & $3(20.0)$ & $174(56.9)$ & $17,330(36.9)$ & 3 & $5,751(5,078-6,501)$ & 174 & $20(12-95)$ \\
\hline Yes & $12(80.0)$ & $132(43.1)$ & $29,614(63.1)$ & 12 & $1,832(492-3,880)$ & 132 & $166(35-360)$ \\
\hline \multicolumn{8}{|c|}{$\begin{array}{l}\text { Patient reminders are handled by the radiology } \\
\text { department }\end{array}$} \\
\hline No & $7(46.7)$ & $150(49.0)$ & $18,894(40.4)$ & 7 & $2,370(395-4,330)$ & 150 & $47(14-213)$ \\
\hline Yes & $8(53.3)$ & $156(51.0)$ & $27,960(59.6)$ & 8 & $3,561(1,081-5,415)$ & 156 & $48(13-295)$ \\
\hline \multicolumn{8}{|l|}{$\begin{array}{l}\text { Multiple reminders to pateint via } \\
\text { mail/phone/EHR/e-mail }\end{array}$} \\
\hline None & $5(33.3)$ & $176(57.5)$ & $15,355(32.7)$ & 5 & $3,429(340-5,078)$ & 176 & $19(12-75)$ \\
\hline $1-2$ & $6(40.0)$ & $87(28.4)$ & $14,382(30.6)$ & 6 & $1,995(589-4,330)$ & 87 & $138(24-293)$ \\
\hline $3-4$ & $4(26.7)$ & $43(14.1)$ & $17,207(36.7)$ & 4 & $3898(1809-6795)$ & 43 & $355(157-543)$ \\
\hline \multicolumn{8}{|l|}{ Risk assessment tool(s) used } \\
\hline No & $8(53.3)$ & $212(69.3)$ & $23,628(50.3)$ & 8 & $2900(957$ - 4704) & 212 & $22(12-144)$ \\
\hline Yes & $7(46.7)$ & $94(30.7)$ & $23,316(49.7)$ & 7 & $2044(589-5751)$ & 94 & $177(41-340)$ \\
\hline \multicolumn{8}{|l|}{ Risk calculator embedded in EHR } \\
\hline No & $11(73.3)$ & $255(83.3)$ & $37,613(80.1)$ & 11 & $3,429(395-5,751)$ & 255 & $31(12-242)$ \\
\hline Yes & $4(26.7)$ & $51(16.7)$ & 9,331 (19.9) & 4 & $1,832(1,104-3,562)$ & 51 & $176(47-293)$ \\
\hline \multicolumn{8}{|c|}{ Interactive patient portal use* ( $\%$ of patients using) } \\
\hline$\leq 50 \%$ & $9(64.3)$ & $221(79.5)$ & $31,078(74.3)$ & 9 & $2,370(1,573-5,751)$ & 221 & $31(13-194)$ \\
\hline$>50 \%$ & $5(35.7)$ & $57(20.5)$ & $10,787(25.8)$ & 5 & $2,414(589-3,429)$ & 57 & $125(13-363)$ \\
\hline
\end{tabular}

Missing (N): interactive patient portal use (1 facility; 28 PCPs; 5079 women)

PROSPR population-based research optimizing screening through personalized regimens, PCP primary care physician, EHR electronic health record

system level (Fig. 1). No specific PCP-level characteristics were found to be statistically significant determinants of screening rates.

\section{DISCUSSION}

This study provided a unique view into multi-level influences on breast cancer screening in primary care settings, and examined a novel set of process-of-care and structural measure in relation to breast cancer screening, overall and by specific age groups that correspond to national screening guidelines. We found only modest effects for structural and process measures for practices as related to breast cancer screening. However, these measures did not seem to impact the proportion of women screened overall, or by guideline-specific age groups.
Woman-level characteristics, such as age, race/ethnicity, insurance, number of primary care visits, and comorbidities were significantly associated with the screening patterns, as has been reported previously $(8,16,17)$. Importantly, this study is the first to estimate the relative influence on breast cancer screening across multiple levels (PCPs, practices, and health system) when accounting for individual patient-level characteristics. We found that for breast cancer screening overall, PCPs and health systems account for most of the variance. Unexplained differences in the percent screened for the age group with the most guideline heterogeneity (40-49year-olds) were predominantly attributed to the health system level, but for screening in the age group with the most guideline consensus (ages 50-74), the providers, followed by practices, accounted for most of the variation. However, variation in screening in the older ages (75+), for which there are no 
Table 3 Percent (\%) Screened and 95\% CIs for Primary Care Practice Screening Processes Adjusted for Woman and Primary Care Provider (PCP) Characteristics

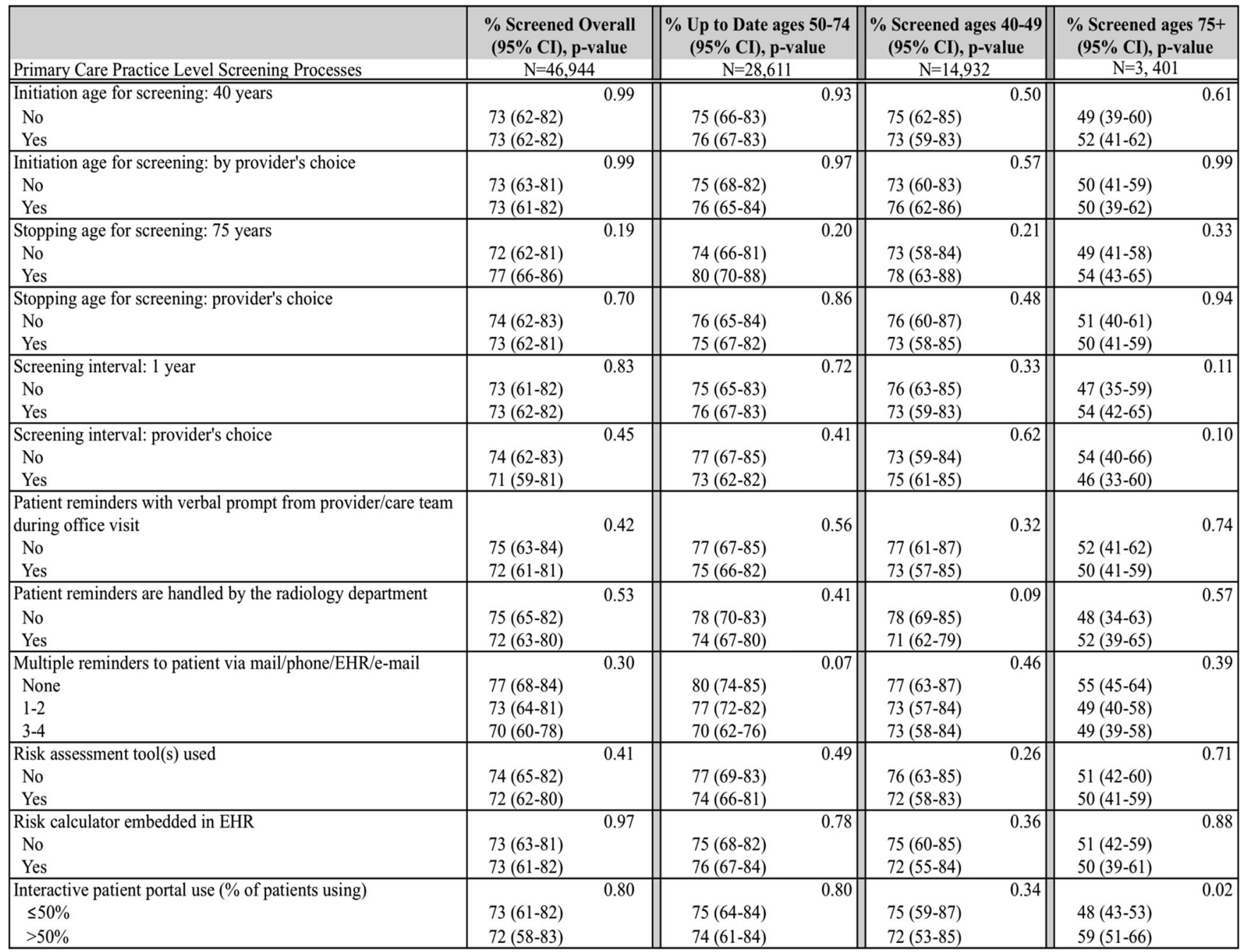

*Adjusted for primary care provider and women characteristics

CI confidence interval, EHR electronic health record

clear guidelines, was also mostly attributable to PCPs. The PCP level seemed to most consistently account for notable variation in breast cancer screening compared to practices and health systems. These findings lend support to targeted interventions at multiple levels, but perhaps with an emphasis at the PCP level.

This study builds upon conceptual models for cancer screening that include processes of care as integral components of the models $(1,3-5)$, and on prior work examining specific process measures. Most of the evidence to date on the role of process measures in cancer screening has been at the provider level. Schapira et al. surveyed PCPs $(N=385)$ and found significant variation in use of breast cancer risk assessment tools, EHR decision support, and use/type of patient reminders for routine screening (7). These differences were related to practice characteristics, particularly National Committee for Quality Assurance (NCQA) recognition as a patientcentered medical home $(\mathrm{PCMH})$. At the same time, structural and process measures studied at the level of radiology practices, have shown significant associations with breast screening outcomes. For example, Taplin et al. reported an association of screening outcomes with facility structural characteristics such as provider composition, affiliation with an academic medical center, and practice size, as well as the interpretive processes of the number of radiologists per mammogram, use of double reading, and other radiology-specific processes (18). Our results underscore the multi-level aspect of existing conceptual models for screening $(1,3,5)$ in that woman, provider, practice, and system effects influence breast screening behaviors.

The Zapka et al. model suggested potential multi-level factors associated with screening outcomes, such as clinician knowledge, incentives, and time at the provider level; outreach/communication methods; medical record system; and patient education resources at the practice and system levels. (5) We were not able include all of these potential structural and process factors in our study, but those we did 
Table 4 Percent Screened and 95\% CIs for Woman and Primary Care Provider (PCP) Characteristics

\begin{tabular}{|c|c|c|c|c|}
\hline $\begin{array}{l}\text { Primary Care Provider and Women } \\
\text { Charateristics* }\end{array}$ & $\begin{array}{l}\text { \% Screened Overall } \\
(95 \% \mathrm{CI}), \mathrm{p} \text {-value }\end{array}$ & $\begin{array}{c}\text { \% Up to Date, } \\
\text { ages } 40-49 \\
(95 \% \mathrm{CI}), \mathrm{p} \text {-value }\end{array}$ & $\begin{array}{c}\text { \% Screened, } \\
\text { ages } 50-74 \\
(95 \% \mathrm{CI}), \mathrm{p} \text {-value }\end{array}$ & $\begin{array}{c}\% \text { Screened, } \\
\text { ages } 75+ \\
(95 \% \mathrm{CI}), \mathrm{p} \text {-value }\end{array}$ \\
\hline \multicolumn{5}{|l|}{ Primary Care Provider $(\mathrm{N}=306)$} \\
\hline Provider Type & 0.08 & 0.02 & 0.11 & 0.29 \\
\hline Physician & $73(64-81)$ & $74(60-85)$ & $76(69-82)$ & $51(47-56)$ \\
\hline Nurse practioner/Physician assistant & $70(57-80)$ & $71(54-84)$ & $71(60-80)$ & $46(29-65)$ \\
\hline Unknown & $68(56-78)$ & $65(48-79)$ & $71(60-79)$ & $41(29-54)$ \\
\hline \multicolumn{5}{|l|}{$\begin{array}{l}\text { Women aged 40+ in Primary Care } \\
(\mathrm{N}=46,944)\end{array}$} \\
\hline Age (years) & $<0.0001$ & \multirow{4}{*}{ NA } & \multirow{4}{*}{ NA } & \multirow{4}{*}{ NA } \\
\hline $40-49$ & $72(63-80)$ & & & \\
\hline $50-74$ & $76(66-83)$ & & & \\
\hline $75+$ & $50(39-61)$ & & & \\
\hline Race/Ethnicity & $<0.0001$ & $<0.0001$ & $<0.0001$ & $<0.0001$ \\
\hline Non Hispanic White & $71(61-80)$ & $72(58-83)$ & $74(66-80)$ & $50(46-55)$ \\
\hline Non Hispanic Black & $78(69-85)$ & $78(64-87)$ & $81(75-86)$ & $52(44-60)$ \\
\hline Non Hispanic Asian/Pacific Islander & $73(63-81)$ & $75(61-86)$ & $75(67-82)$ & $41(28-54)$ \\
\hline Hispanic & $86(80-91)$ & $86(76-92)$ & $89(84-92)$ & $66(56-75)$ \\
\hline Other/Unknown & $76(66-83)$ & $76(62-86)$ & $79(72-85)$ & $44(33-56)$ \\
\hline Insurance & $<0.0001$ & $<0.0001$ & $<0.0001$ & $<0.0001$ \\
\hline Private & $75(65-82)$ & $76(62-86)$ & $77(70-83)$ & $55(48-61)$ \\
\hline Medicaid & $64(53-74)$ & $63(47-77)$ & $69(59-77)$ & $34(18-54)$ \\
\hline Medicare & $71(61-79)$ & $69(53-81)$ & $73(66-80)$ & $50(46-55)$ \\
\hline Other $^{T}$ & $74(64-82)$ & $73(58-85)$ & $77(69-83)$ & $47(35-61)$ \\
\hline Uninsured & $65(54-75)$ & $63(47-77)$ & $69(60-76)$ & $49(40-59)$ \\
\hline $\begin{array}{l}\text { Number of primary care provider visits in } \\
\text { calendar year }\end{array}$ & $<0.0001$ & $<0.0001$ & $<0.0001$ & $<0.0001$ \\
\hline 0 & $69(50-83)$ & $75(35-95)$ & $72(52-86)$ & $38(5-87)$ \\
\hline 1 & $72(62-80)$ & $67(52-80)$ & $69(61-76)$ & $41(36-47)$ \\
\hline 2 & $76(66-83)$ & $73(59-84)$ & $75(67-81)$ & $50(45-56)$ \\
\hline $3-4$ & $79(71-86)$ & $77(64-86)$ & $78(71-84)$ & $52(47-57)$ \\
\hline $5+$ & $69(50-83)$ & $82(71-90)$ & $81(75-86)$ & $55(50-60)$ \\
\hline Comorbidities index & $<0.0001$ & $<0.0001$ & $<0.0001$ & $<0.0001$ \\
\hline None & $75(65-82)$ & $75(61-85)$ & $77(70-83)$ & $54(49-58)$ \\
\hline One & $73(63-81)$ & $74(60-85)$ & $75(68-82)$ & $50(44-55)$ \\
\hline Two or more & $61(50-71)$ & $61(44-75)$ & $63(54-71)$ & $44(39-50)$ \\
\hline
\end{tabular}

CI confidence interval, $P C P$ primary care provider

*Missing (N): racelethnicity (225), insurance (1278), comorbidity score (664)

tOther insurance defined as other federal or state health insurance programs or not otherwise specified

include (outreach/communication methods, medical record system, and appointment times) were not found to be associated with the percent of women screened. Examining a fuller range of structural and process measures in a broad sample of health systems is warranted for understanding screening outcomes.

Given the provider-reported heterogeneity in practice-level process systems in the Schapira et al. study (7), and our finding of a preponderance of unexplained variation at the PCP level, one interpretation is that the high degree of PCP-attributed variation in breast cancer screening may best be mitigated by practice-level interventions. Practices could make actionable targets for interventions with the potential to "rein in" the PCP-level variation. However, it is important to note that some policies and preferred patterns of care may be decided at the health system level, not the practice level.
Building on prior evidence addressing PCP and radiology facility processes of care, our study helps to fill knowledge gaps regarding the PCP- and practice-level variation in breast cancer screening utilization and patterns of care. Expanding the evidence related to multilevel processes of care is a critical step in identifying appropriate targets for interventions. A recent study that quantified variation in screening outcomes for breast, cervical, and colorectal cancer across the PROSPR consortium underscored the need for multi-level analyses investigating patient, healthcare provider, and health system characteristics, as significant variation at the levels of clinic and healthcare system was associated with timely follow-up (19). Potential interventions to increase use of effective care are likely to be at both the practice level, 


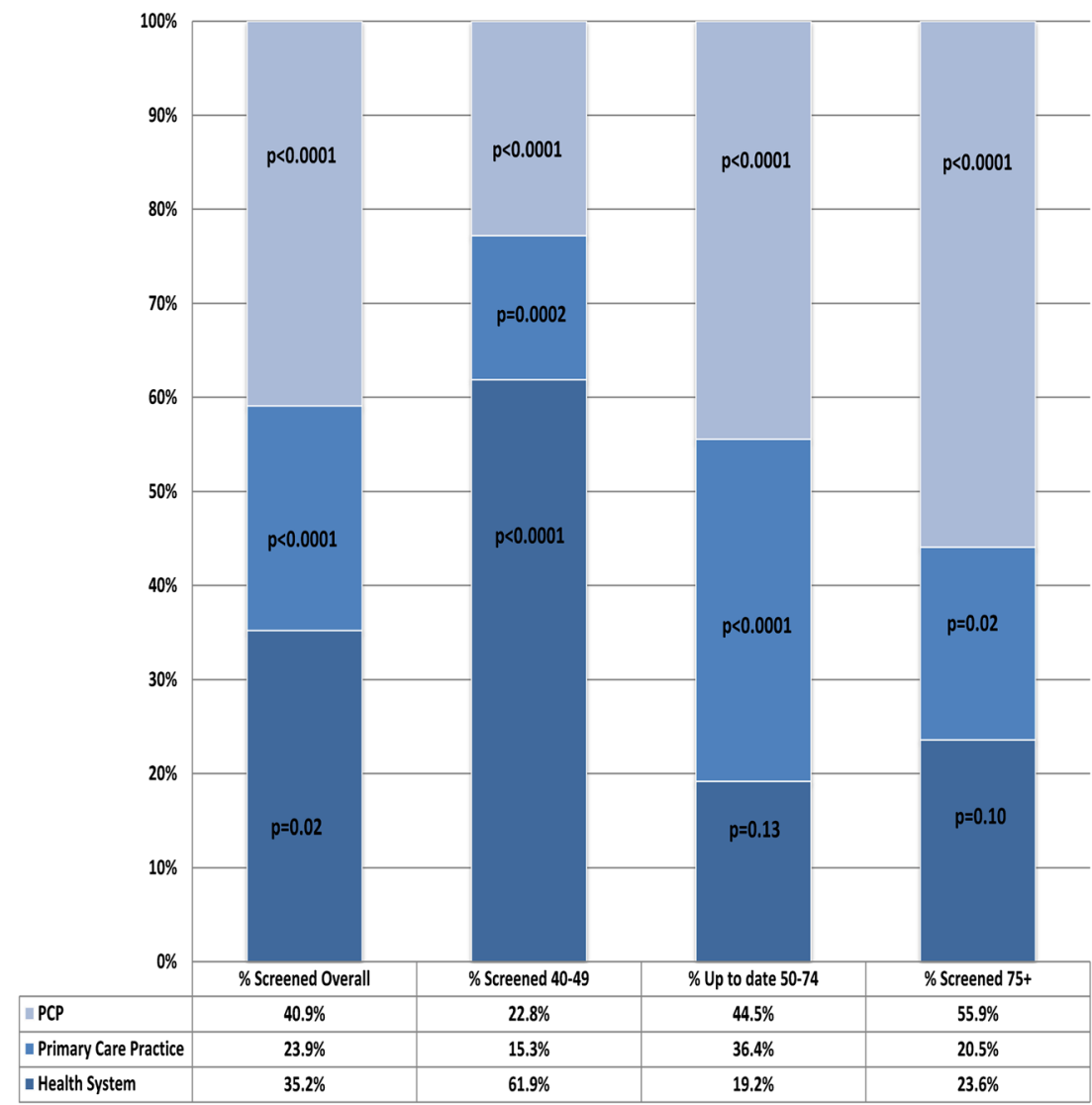

Figure 1 Summary of variance components analysis for screening outcomes at the primary care provider (PCP), primary care practice and health system levels.

such as practice alerts (to trigger action during visits), and health system level, such as registries (for outside of visits/population health management).

While our study has the strengths of robust multi-level analyses and novel process measures, it was limited in several ways. First, although the total number of women represented (almost 47,000) was respectable, the number of primary care clinics included would ideally have been larger to potentially capture more health system diversity. Second, the PCP-level characteristics we included were somewhat limited because we only included those with complete data. In addition, because we had a fully nested, hierarchical design (i.e., women nested within PCPs, who were nested within practices), we only included PCPs who were affiliated with practices that fully responded to the survey. Potentially important PCP characteristics might have included age, gender, and years in practice, but were beyond the scope of this study. We recognize that inclusion of radiology practices could reveal additional sources of variation. Also, we acknowledge the important roles of patient preferences, health beliefs, health literacy, etc. in women's breast screening behaviors.

In conclusion, our findings that the measured primary care processes do not seem to be related to breast cancer screening utilization patterns was somewhat surprising, but highlights the importance of woman-level characteristics in studies of screening use. Further, even after accounting for woman-level characteristics, the remaining variation in breast cancer screening was largely due to PCP and health system levels. Our quantification of variation at multiple levels may help inform the design of targeted intervention strategies to improve the use of breast cancer screening.

Acknowledgements: The authors thank the participating PROSPR Research Centers for the data they have provided for this study. A list of the PROSPR investigators and contributing research staff is provided at: http://healthcaredelivery.cancer.gov/prospr/

Corresponding Author: Martha Goodrich, MS; Department of Biomedical Data Science, Geisel School of Medicine at Dartmouth, Lebanon, NH, USA (e-mail: martha.e.goodrich@Dartmouth.edu).

Funders This project was supported by the National Cancer Institute (NCI) Population-based Research Optimizing Screening through Personalized Regimens (PROSPR) initiative (U54CA163307).

\section{Compliance with Ethical Standards:}

Conflict of Interest: The authors declare that they do not have a conflict of interest.

Financial Disclosure: No financial disclosures were reported by the authors of this paper. 


\section{REFERENCES}

1. Beaber EF, Kim JJ, Schapira MM, Tosteson AN, Zauber AG, Geiger AM, et al. Unifying screening processes within the PROSPR consortium: a conceptual model for breast, cervical, and colorectal cancer screening. J Natl Cancer Inst. 2015;107(6):djv120.

2. Breslau ES, Jeffery DD, Davis WW, Moser RP, McNeel TS, Hawley S. Cancer screening practices among racially and ethnically diverse breast cancer survivors: results from the 2001 and 2003 California health interview survey. J Cancer Surviv. 2010;4(1):1-14.

3. Onega T, Haas JS, Bitton A, Brackett C, Weiss J, Goodrich M, et al. Alignment of breast cancer screening guidelines, accountability metrics, and practice patterns. Am J Manag Care. 2017;23(1):35-40.

4. Yabroff KR, Zapka J, Klabunde CN, Yuan G, Buckman DW, Haggstrom D, et al. Systems strategies to support cancer screening in U.S. primary care practice. Cancer Epidemiol Biomark Prev. 2011;20(12):2471-9.

5. Zapka JG, Puleo E, Taplin SH, Goins KV, Ulcickas Yood M, Mouchawar $\mathbf{J}$, et al. Processes of care in cervical and breast cancer screening and follow-up-the importance of communication. Prev Med. 2004;39(1):8190.

6. Chang CH, Bynum JP, Onega T, Colla CH, Lurie JD, Tosteson AN. Screening mammography use among older women before and after the 2009 U.S. Preventive Services Task Force Recommendations. J Womens Health (Larchmt). 2016;25(10):1030-7.

7. Schapira MM, Sprague BL, Klabunde CN, Tosteson AN, Bitton A, Chen JS, et al. Inadequate systems to support breast and cervical cancer screening in primary care practice. J Gen Intern Med. 2016;31(10):114855.

8. Hubbard RA, O'Meara ES, Henderson LM, Hill D, Braithwaite D, Haas JS, et al. Multilevel factors associated with long-term adherence to screening mammography in older women in the U.S. Prev Med. 2016;89: 169-77.

9. Baughman AW, Brawarsky P, Onega T, Tosteson TD, Wang $\mathbf{Q}$, Tosteson AN, et al. Medical home transformation and breast cancer screening. Am J Manag Care. 2016;22(11):e382-e8.
10. Gerrity MS, DeVellis RF, Earp JA. Physicians' reactions to uncertainty in patient care. A new measure and new insights. Med Care. 1990;28(8):724-36.

11. Yano EM, Soban LM, Parkerton PH, Etzioni DA. Primary care practice organization influences colorectal cancer screening performance. Health Serv Res. 2007;42(3 Pt 1):1130-49.

12. Agency for Healthcare Research and Quality. Selecting Process Measures for Clinical Quality Measurement 2016; https://www.qualitymeasures. ahrq.gov/help-and-about/quality-measure-tutorials/selecting-processmeasures accessed on June 4, 2018.

13. Onega T, Beaber EF, Sprague BL, Barlow WE, Haas JS, Tosteson AN, et al. Breast cancer screening in an era of personalized regimens: a conceptual model and National Cancer Institute initiative for risk-based and preference-based approaches at a population level. Cancer. 2014;120(19):2955-64.

14. National Cancer Institute, Division of Cancer Control \& Population Sciences. Population-based Research Optimizing Screening through Personalized Regimens (PROSPR) 2017; https://healthcaredelivery.cancer.gov/prospr/ accessed on June 4, 2018.

15. Klabunde RE. Effects of adenosine on sympathetic vasoconstriction in dog gracilis muscle. J Pharmacol Exp Ther. 1987;240(1):106-10.

16. Henry KA, McDonald $\mathbf{K}$, Sherman $\mathbf{R}$, Kinney AY, Stroup AM Association between individual and geographic factors and nonadherence to mammography screening guidelines. $\mathrm{J}$ Womens Health (Larchmt). 2014;23(8):664-74.

17. Rahman SM, Dignan MB, Shelton BJ. Factors influencing adherence to guidelines for screening mammography among women aged 40 years and older. Ethn Dis. 2003;13(4):477-84.

18. Taplin S, Abraham L, Barlow WE, Fenton JJ, Berns EA, Carney PA, et al. Mammography facility characteristics associated with interpretive accuracy of screening mammography. J Natl Cancer Inst. 2008;100(12):876-87.

19. Tosteson AN, Beaber EF, Tiro J, Kim J, McCarthy AM, Guinn VP, et al Variation in screening abnormality rates and follow-up of breast, cervical and colorectal cancer screening within the PROSPR consortium. J Gen Intern Med. 2016;31(4):372-9. 